\title{
Radiation Efficient Unidirectional Low-Profile Slot Antenna Elements for X-Band Application
}

\author{
C. Löcker, T. Vaupel, and T. F. Eibert \\ FGAN-Research Institute for High Frequency Physics and Radar Techniques (FHR), Neuenahrer Str. 20, \\ 53343 Wachtberg-Werthhoven, Germany
}

\begin{abstract}
Slots in metallic ground planes are very promising candidates for conformal antenna applications. However, a low-profile unidirectional antenna requires a back reflector close to the slot and the resulting stripline feed causes strong excitation of parallel-plate modes. In this contribution, we consider unidirectional reflector-backed slot configurations with parallel-plate mode suppression by shorting pins. Starting from a parametric study with respect to shorting pin location and back reflector distance, we present a stripline-fed rectangular slot element with radiation efficiency of more than $80 \%$ and a bandwidth of about $5 \%$ at centre frequency $10 \mathrm{GHz}$. A careful optimisation of shorting pin locations guarantees reliable parallel-plate mode suppression without deteriorating the slot radiation behaviour. Coupling coefficients between parallel and aligned rectangular slot elements are presented. For increased bandwidth applications, a bowtie slot element with about $8 \%$ bandwidth and radiation efficiency of close to $80 \%$ is proposed.
\end{abstract}

\section{Introduction}

Due to their low profile, light weight, robustness and low cost, antennas in printed circuit technology are widely used in electronic warfare, communication, automotive, etc. Traditionally, many design instructions can be found in literature for patch antennas (James et al., 1981). However, due to its resonant behaviour the radiating patch element has only narrow bandwidth, unless thick substrates are used, which is disadvantageous with respect to integration into non-planar surfaces. The surrounding of the patch is inherently dielectric, which benefits propagation of surface waves resulting in low radiation efficiency.

The radiating slot element is an attractive alternative. A slot in a metallic ground plane is the complementary structure of the corresponding electric dipole. Since the radiation pattern of the slot is bidirectional, a back reflector must be used to avoid back radiation. With respect to antenna integration in curved surfaces, the reflector should

Correspondence to: C. Löcker

(loecker@fgan.de) be placed closely to the slot, resulting in a typical stripline or triplate configuration. Unfortunately, this structure admits parallel-plate propagation and the discontinuity slot/stripline excites the corresponding modes. Higher order modes can be avoided by choosing a thin substrate, because their cut-off frequencies increase by decreasing the distance between the ground planes. The suppression of the fundamental mode, however, is a challenging task, since it is a TEM type mode starting at $0 \mathrm{~Hz}$. One method to suppress the TEM mode is the use of twin slot configurations for destructive interference of the excited waves. An alternative method is the cavity backed slot (Galejs, 1963; Biebl and Friedsam, 1995; Omiya et al., 1998). Metallic walls force the electric field of the TEM wave to zero. A similar procedure is the placement of shorting pins around the slot aperture, connecting the slot's ground plane with the back reflector (Sommers, 1955). Compared to cavity backed slots, only little information about guidelines on positioning of vias is available in literature. Some papers treat stipline-slot-fed patch antennas (Brachat and Baracco, 1995; Bhattacharyya et al., 1998), where a theoretical analysis for via-slot interaction is given in the latter.

In this contribution, a parametric study is concerned with the influence of via and reflector positions on the input impedance and radiation efficiency of a single rectangular slot element. Then, an optimised unidirectional slot element printed on a very thin substrate with parallel-plate mode suppression by shorting pins is presented by simulated and measured data, based on the approach proposed in (Sommers, 1955). Towards array applications, we present mutual coupling investigations for different slot arrangements. A second radiating bow-tie like aperture for bandwidth enhancement is presented by numerical and experimental results.

\section{Rectangular Slot Element}

The geometry of the investigated rectangular slot element is depicted in Fig. 1. The slot is located in the upper ground plane and is excited by a stripline located in the centre of the substrate with $\epsilon_{r}=2.2$. In order to suppress the parasitic parallel plate mode, the upper and lower ground planes are 


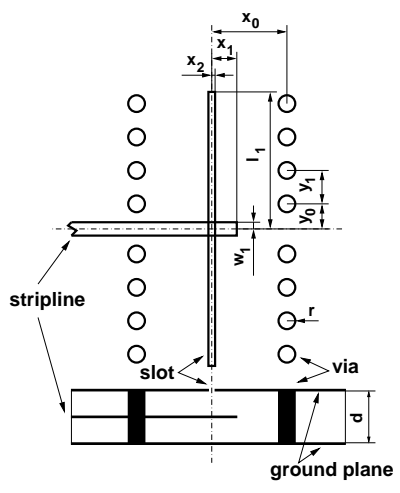

(a)

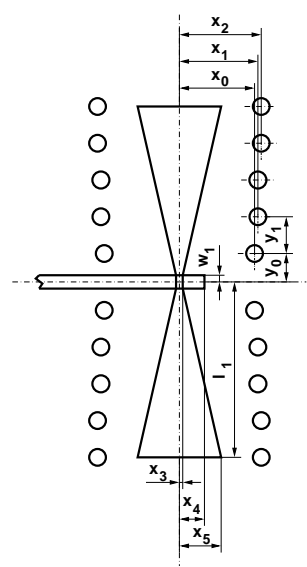

(b)
Fig. 1. Geometries of investigated apertures: (a) stripline-fed rectangular slot element, (b) bow-tie slot element.

connected by shorting pins or vias. Antenna dimensions are (unit: millimetre): $x_{0}=4.5, x_{1}=1.5, x_{2}=0.25, y_{0}=1.5, y_{1}=2.2$ (constant distance in y-direction), $r=0.5, d=1, w_{1}=0.435$ and $l_{1}=8.25$.

To gain a broad understanding of the influence of via positions and reflector distances on the resonance behaviour, the slot was investigated by a parametric study. To avoid transformation and compensation effects by coupling from the slot to the stripline and the open stripline stub, the stripline was removed and the slot was excited by a magnetic $\delta$-gap voltage source. The terminal impedance was computed by a surface/volume integral equation method for the analysis of planar circuits with additional 3-D-components embedded in a multilayered medium of infinite extent (Vaupel and Hansen, 1999; Vaupel et al., 2003). Thus, the layered medium environment is analytically considered by its spectral domain Green's function and only the magnetic surface currents in the slot aperture as well as the electric via currents (modelled as volume currents) must be discretised.

Numerical results on the input impedance and radiation efficiency show Figs. 2, 3 and Figs. 4, 5, respectively, with respect to via-slot distance $x_{0}$ and back reflector-slot distance $d$. The curves for the real and imaginary part of the input impedance show a typical resonator behaviour. The reflector and the vias cause a shift of the input impedance to the inductive regime. The frequency of the first resonance (corresponding to the peak input resistance) increases and the peak resistance decreases and broadens with decreasing via and reflector distance, resulting in a reduced effective length of the slot. The efficiency curves show that the parallel plate mode can be effectively suppressed by the vias. At the frequency band edges, Fig. 4 shows a decreasing efficiency for vias placed too far as well as too close to the slot. A distance $x_{0}$ smaller than approx. $0.25 \lambda$ should ensure efficiencies over $90 \%$. If the back reflector is too close to the slot, the parallel plate mode is excited stronger, yielding a lower efficiency.

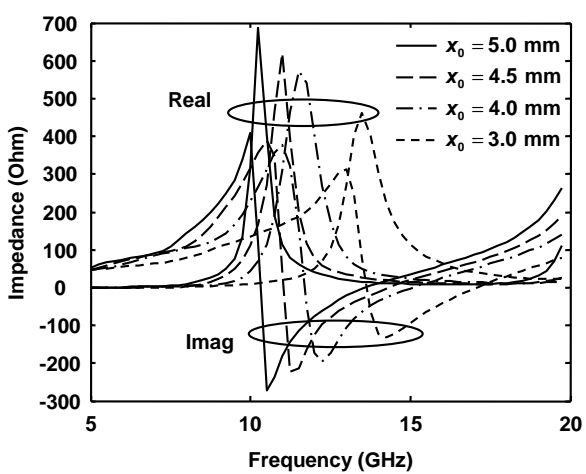

Fig. 2. Parametric study: Input impedance with respect to viaposition $x_{0}$.

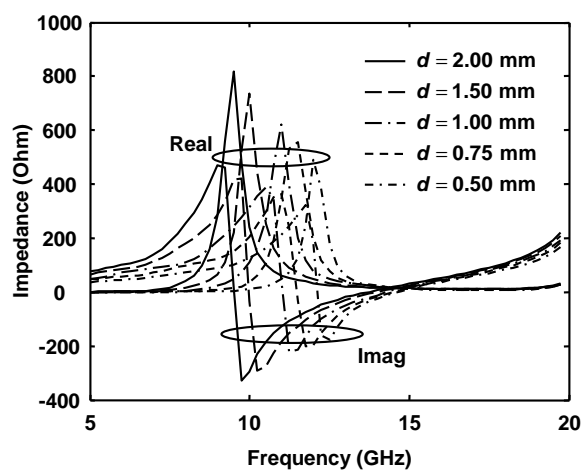

Fig. 3. Parametric study: Input impedance with respect to reflector distance $d$.

The fabricated single slot element with dimensions as described in Fig. 1 is excited by a stripline, which is open at its end. Thus, the stub can be used to optimise impedance matching. The slot was printed on a $90 \mathrm{~mm} \times 90 \mathrm{~mm}$ Rogers 5880 Duroid substrate and should have a theoretical directivity of about $5.15 \mathrm{~dB}$ (gain of a lossless $\lambda / 2$-dipole increased by $3 \mathrm{~dB}$ due to missing back radiation). In order to decrease the number of degrees of freedom, the width of the feeding stripline was chosen for $50 \Omega$. The slot's dimensions were carefully optimised by Agilent's ADS2003A Momentum (ADS), including metallisation (copper) and dielectric losses. The directivity was estimated by integrating the radiation fields over all radiation directions and the gain is determined by the gain transfer method. A slightly higher resonance frequency was chosen in ADS to obtain a resonance frequency of $10 \mathrm{GHz}$. Figure 6 depicts simulated (ADS) and measured return loss data. The measured bandwidth (return loss $<-10 \mathrm{~dB}$ ) is about $5 \%$. Simulated and measured data are in good agreement. The efficiency curve in Fig. 7 indicates, that the efficiency does not decrease below about $80 \%$ in the band of operation. 


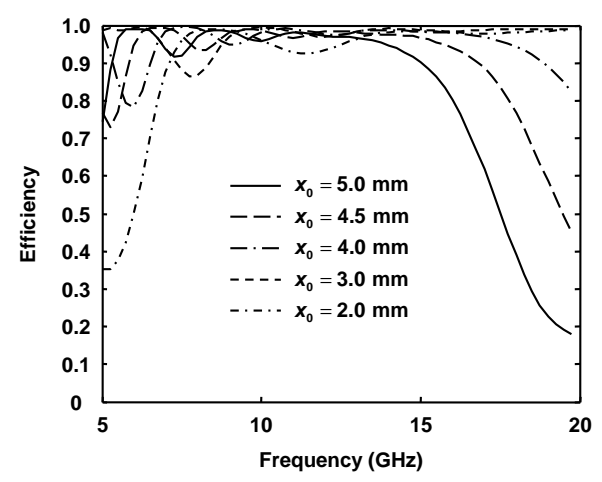

Fig. 4. Parametric study: Efficiency with respect to via-position $x_{0}$.

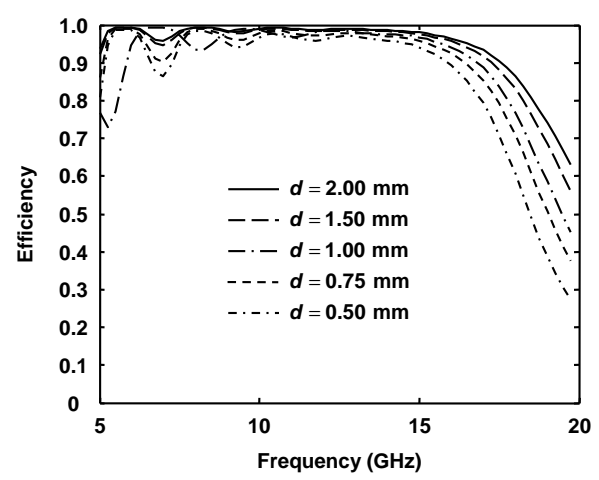

Fig. 5. Parametric study: Efficiency with respect to reflector distance $d$.

\section{Mutual coupling between rectangular slot elements}

Mutual coupling between antenna elements is an important criterion in conformal array antenna applications. We investigated collinear and broadside slot arrangements. Three slots were considered in each configuration. The single slot dimensions are taken from Fig. 1a. In the collinear configuration, centre-centre distances with respect to the first slot are $22.5 \mathrm{~mm}$ and $52.5 \mathrm{~mm}$. In the broadside slot configuration, the slots could be arranged closer to each other, resulting in separations of $15 \mathrm{~mm}$ and $45 \mathrm{~mm}$. The mutual coupling was determined by transmission measurements between neighbouring slots with a HP8510 network analyser. The remaining third port was terminated by a $50 \Omega$ match. Figures 8 and 9 show simulated and measured scattering coefficients $S_{i j}$, which describe the coupling behaviour. For collinear alignment the radiation null in the corresponding direction causes a smaller coupling compared to the coupling for broadside configuration. In this case, the radiated space wave travelling along the slot ground plane creates the relative large coupling. By placing a metallic plate between the slot aperture, it can be demonstrated that the mutual coupling due to parallel-plate mode propagation is effectively suppressed by the vias.

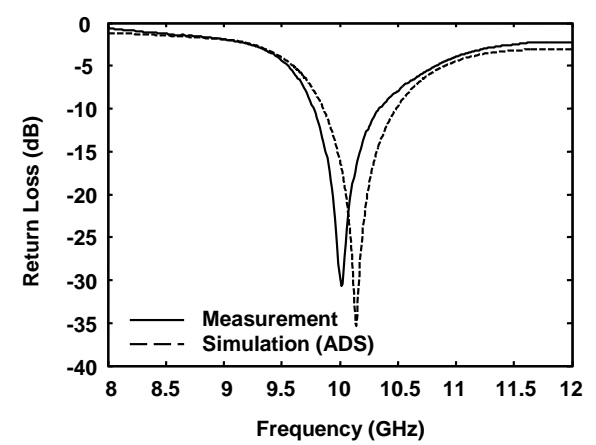

Fig. 6. Rectangular slot element, simulated and measured return loss.

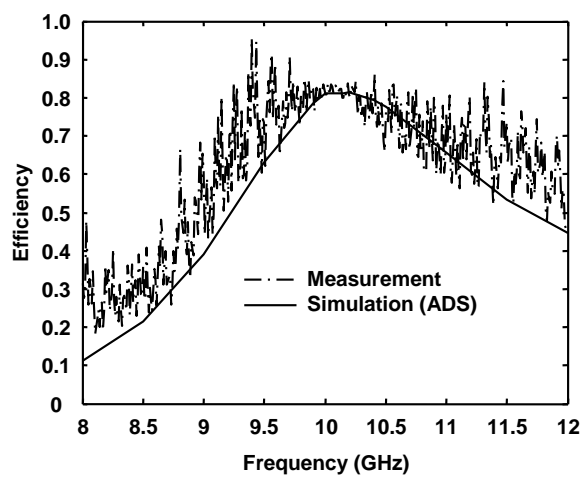

Fig. 7. Rectangular slot element, simulated and measured efficiency.

\section{Bow-Tie Slot Element}

Since the bandwidth of the rectangular slot element is relatively small, we also investigated bow-tie like slot elements (Kraus, 1988). The slot was widened at its ends and the length was increased to about $0.7 \lambda_{0}$ for a centre frequency of $10 \mathrm{GHz}$, see Fig. 1. In order to reduce edge diffraction effects, the side length of the substrate was increased to $100 \mathrm{~mm}$. In this structure the vias positions are not parallel to the aperture axis. The vias positions were carefully optimised to achieve the design requirements. The bowtie dimensions are (units: millimetre): $x_{0}=4.25, x_{1}=4.45$, $x_{2}=4.65, x_{3}=0.25, x_{4}=1.6, x_{5}=2.5, y_{0}=1.7, y_{1}=2.2, w_{1}=0.425$ and $l_{1}=10.5$. As for the rectangular slot element, the substrate has an permittivity of 2.2. Figure 10 depicts the measured and simulated return loss. The $10 \mathrm{~dB}$ bandwidth amounts to $0.8 \mathrm{GHz}$ and is higher than for the rectangular slot element. The measured antenna efficiency is about $75-80 \%$, see Fig. 11, in the band of operation.

\section{Conclusion}

Radiating slots in a metallic ground plane were investigated. The upper ground plane was connected with a back reflector 


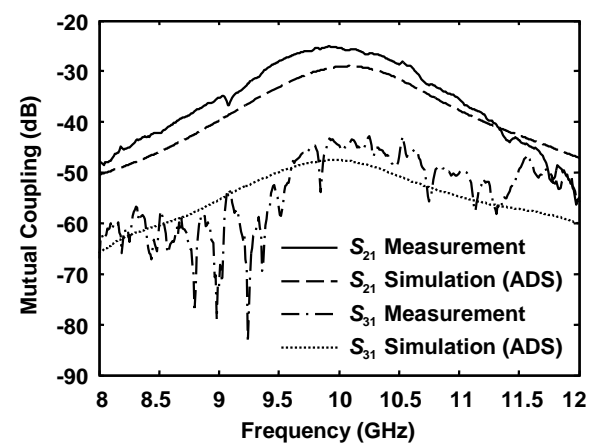

Fig. 8. Mutual coupling, collinear slot arrangement.

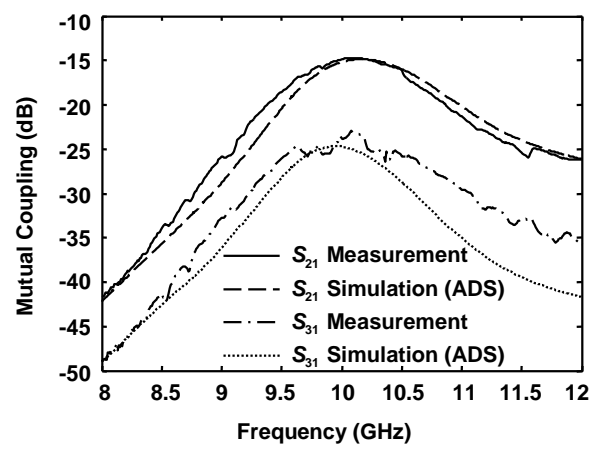

Fig. 9. Mutual coupling, broadside slot arrangement.

by shorting pins. It is shown by simulation and measurement, that the parasitic TEM parallel plate mode can be effectively suppressed by shorting pins. However, the slot design must pass a careful optimisation process, because of the strong influence of the vias on the resonance and broadband behaviour. A stripline-fed rectangular slot element with a $10 \mathrm{~dB}$-bandwidth of $5 \%$ at centre frequency of $10 \mathrm{GHz}$ and an efficiency of more than $80 \%$ was fabricated. Studies on coupling effects between aligned slots show that collinear slots are non-critical. For bandwidth enhancement, a bow-tie like slot element with $10 \mathrm{~dB}$ bandwidth of $8 \%$ and radiation efficiency of close to $80 \%$ was presented.

\section{References}

Bhattacharyya, A., Fordham, O., and Liu, Y.: Analysis of StriplineFed Slot-Coupled Patch Antennas with Vias for Parallel-Plate Mode Suppression, IEEE Transactions on Antennas and Propagation, 46, 538-545, 1998.

Biebl, E. M. and Friedsam, G. L.: Cavity-Backed Aperture Antennas with Dielectric and Magnetic Overlay, IEEE Transactions on Antennas and Propagation, 43, 1226-1232, 1995.

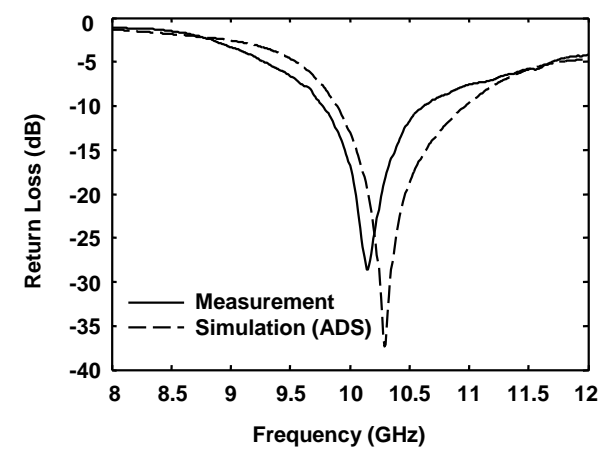

Fig. 10. Bow-Tie element, measured and simulated return loss.

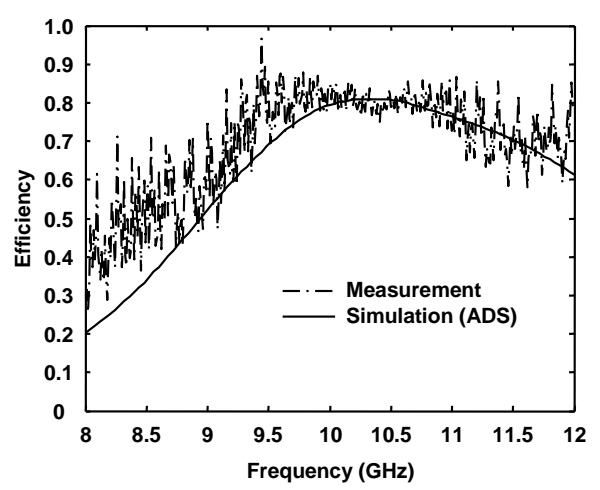

Fig. 11. Bow-Tie element, measured and simulated efficiency.

Brachat, P. and Baracco, J. M.: Dual-Polarization Slot-Coupled Printed Antennas Fed by Stripline, IEEE Transactions on Antennas and Propagation, 43, 738-742, 1995.

Galejs, J.: Admittance of a Rectangular Slot Which is Backed by a Rectangular Cavity, IEEE Transactions on Antennas and Propagation, 119-126, 1963.

James, J. R., Hall, P. S., and Wood, C.: Microstrip Antenna Theory and Design, Peter Peregrinus Ltd., 1981.

Kraus, J. D.: Antennas, McGraw Hill, 1988.

Omiya, M., Hikage, T., Ohno, N., Horiguchi, K., and Itho, K.: Design of Cavity-Backed Slot Antennas Using the Finite-Difference Time-Domain Technique, IEEE Transactions on Antennas and Propagation, 46, 1853-1858, 1998.

Sommers, D. J.: Slot Array Employing Photoetched Tri-Plate Transmission Lines, IEEE Transactions on Microwave Theory and Techniques, 3, 157-162, 1955.

Vaupel, T. and Hansen, V.: Electrodynamic Analysis of Combined Microstrip and Coplanar/Slotline Structures with 3-D Components Based on a Surface/Volume Integral Equation Approach, IEEE Transactions on Microwave Theory and Techniques, 47, 1788-1800, 1999.

Vaupel, T., Hansen, V., and Schäfer, F.: Radiation Efficiency Analysis of Submm-Wave Receivers Based on a Modified Spectral Domain Technique, Radio Sience, 4, 13-1-13-2, 2003. 